\title{
NUEVOS TESTIMONIOS ARQUEOLÓGICOS Y EPIGRÁFICOS DE MEDINA DE LAS TORRES (BADAJOZ), EN UN MANUSCRITO INÉDITO DEL SIGLO XIX
}

\author{
NEW ARCHAEOLOGICAL AND EPIGRAPHIC EVIDENCE FROM MEDINA DE LAS \\ TORRES (BADAJOZ), FROM A XIXTH CENTURY UNPUBLISHED MANUSCRIPT
}

\author{
por \\ H. GIMENO PASCUAL y J.L. RAMÍREZ SÁDABA
}

\begin{abstract}
RESUMEN En este trabajo se dan a conocer nuevos datos epigráficos y topográficos contenidos en un manuscrito de 1845 de José Barrientos, corresponsal de la Comisión de Monumentos de Badajoz, que inciden en la localización del municipio de Contributa Iulia en el cerro de Los Cercos, en las cercanías de Medina de las Torres (Badajoz). Además, se edita el primer testimonio de Hispania de una dedicación a las Matres Augustae por unos Seviri Primi.
\end{abstract}

ABSTRACT New epigrafic and topografic data are given to know in this report, which fall into siting Contributa Iulia in Los Cercos's hill, near to Medina de las Torres (Badajoz). They are contained into a manuscript from 1845 of José Barrientos correspondent of Comisión de Monumentos de Badajoz and it has the first testimony of Hispania about a dedication by a Seviri Primi to the Matres Augustae.

Hasta el siglo XIX la localidad de Medina de las Torres (Badajoz), arqueológicamente o como sitio antiguo, era prácticamente desconocida. Rodrigo Caro fue uno de los pioneros en transmitir inscripciones de dicha localidad que se conservaban reutilizadas en diversos edificios, junto con Tamayo de Salazar ${ }^{1}$. A pesar de este número relativamente importante de inscripciones Medina de las Torres no despertó ningún interés por parte de la historiografía local debido, probablemente, a que no conservaba visible ningún resto arquitectónico o escultórico.

El panorama cambiaría a mediados del siglo XIX. Gracias a la instauración de la Comisión Provincial de Monumentos Históricos y Artísticos de Badajoz y a las visitas de sus corresponsales a los pueblos, conservamos las noticias de algunos hallazgos arqueológicos que se habían producido al final de la década

1. CIL II 1025 en la ermita de San Blas (San Bartolomé, según Alsinet); CIL II 1026 y CIL II 1031-1032, en la iglesia parroquial, donde también sitúa Tamayo de Salazar CIL II 1029. Por último, CIL II 984, para la que Caro da como procedencia Zafra; Masdeu y Constanzo (Constanzo al menos da medidas), sin embargo, también la sitúan en Medina de las Torres. 
de los 40 de dicho siglo. Además de lo que contiene el manuscrito que estudiamos, en 1845 se hallaron tres estatuas romanas en la Dehesa del Castillejo ${ }^{2}$, trozos de columnas, y otros fragmentos "de antigüedad" que fueron custodiados en el Ayuntamiento. A partir de entonces Medina de las Torres entraría en la nómina de lugares antiguos a preservar y excavar-aunque desafortunadamente aún no se ha llevado a la práctica ${ }^{3}$-. Tanto es así que, en 1877, la Comisión de Monumentos de Badajoz hace una visita a Medina para verificar las condiciones en que eran custodiadas las estatuas halladas en 1845 (BB. AA. 97-5/6) y, además, en un informe de 8 de enero de 1896 firmado en Badajoz por el Vicepresidente-Fernando Bernáldez-y el secretario -Tomás Romero de Castilla- de la Comisión de Monumentos Históricos y Artísticos de dicha provincia sobre los despoblados de Badajoz donde deben hacerse excavaciones (AGA: caja 6769), ya se menciona entre ellos el “... despoblado cerca de Medina de las Torres en la dehesa mencionada de los Cercos. Se cree que tuvo alli asiento la antigua Perceiana. En el año 1848 fueron descubiertos en aquel punto tres estatuas colosales, trozos de columnas, y otros fracmentos de antigüedad; por acuerdo de la Comisión están depositados estos objetos en dicho pueblo bajo la custodia del Alcalde hasta tanto que se pueda disponer de fondos para trasladarlas a este Museo provincial".

Quizá no sea ninguna exageración pensar que la causa de esta entrada fuera una memoria sobre el término de Medina de las Torres del año 1845, elaborada por José Barrientos que ha permanecido inédita y donde se hace la primera relación de los hallazgos del término. Este manuscrito se conserva en la Academia de Bellas Artes de San Fernando de Madrid (Archivo. Biblioteca 44-4 / 2) adjunto a un oficio con fecha de 17 de octubre de 1845 de la Comisión de Monumentos Históricos y Artísticos de la provincia de Badajoz, remitido al Ministro de la Gobernación de la Península y Presidente de la Comisión General. El 21 de octubre del mismo año, el subdirector del negociado $3^{\circ}$ de la Sección de Instrucción Pública del Ministerio de la Gobernación, lo remite a la Comisión Central de Monumentos que, con fecha 29 de octubre, ordena que pase al expediente con extracto para que informe la sección.

El firmante del oficio, con fecha 17 de octubre de 1845, remite un extracto de la Memoria de D. José Antonio Barrientos ${ }^{4}$, corresponsal por la provincia de Badajoz, sobre Medina de las Torres, informando al mismo tiempo de las actividades en curso de Barrientos para la Comisión: en breve remitirá nuevas Memorias, y acaso una copia manuscrita de la Historia de esta Provincia que espera adquirir por la cooperación y buenos oficios de la científica comisión de aquella ciudad.

El manuscrito carece de un título particular, tiene nueve folios en los que se incluyen seis dibujos. La letra es la normal en la documentación del siglo XIX y ni paleográfica ni codicológicamente tiene ninguna característica reseñable. En la edición del texto hemos procurado normalizar la grafía a la ortografía actual.

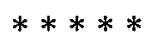

2. BB.AA. 44-4/2. A esta dehesa da nombre el arroyo Castillejo, que es el que bordea la Colina de San Blas, paraje que después se denominaría Los Cercos, término con el cual también hoy se conoce.

3. Aún en el año 1976 R. Rodríguez Bordallo y A.M. Ríos Graña (1976: 159), en el V Congreso de Estudios Extremeños, acaban su comunicación reivindicando medios económicos para la excavación.

4. Como se trata de un extracto de la Memoria es posible que la Memoria sea el documento que se conserva en el Museo de Badajoz y que se halla en estudio por Pablo Ortiz, a quien agradecemos la noticia. Del mismo corresponsal es una memoria sobre Regina, cf. Gimeno 1999. 
Al extractar las noticias y apuntes a que me referiré, sin perder de vista la concisión y laconismo que este trabajo requiere, no he podido dispensarme de fijar algunas razones que depuran el verdadero terreno que ocupó el pueblo romano llamado Percejana; así por consiguiente, creo inutil detenerme en la posición topográfica de Medina de las Torres, que solo por hallarse en su parroquia una piedra sepulcral, ilegible en el día y según la copia sacada en 1824 que acompaño con el $n^{\circ} 1$ se infiere fuese donde existió la población de Contributa Iulia; a poca distancia en el punto que ocupa S. Bartolomé el Real, se encuentran señales mas ciertas, con vestigios de anterior población; y confirma que sean estos escombros los de Contributa, el Itinerario de Antonino, documento que mas noticias nos da de los pueblos del imperio sitos en la Via Militar cuya copia pongo unida con el $n^{\circ}$ 2: mas no creyendo deba fijar ni detener nuestra atención esto que aisladamente aparece, pasemos a lo que reclama mas imperiosamente, trabajos asiduos y constante explotación; las inmensas riquezas que abrigara en su seno, la Colina de San Blas a poco más de 1/4 de legua de aquel, prolongada de $N$ a $S$ y bañadas sus bases por los arroyos de Castillejo y $S$. Blas, es la que nos debe ocupar: rodados escombros nos proporcionan todos los días muestras de la opulenta población romana que debió ostentar en su centro; piedras de granito labradas en figura de ara con inscripciones, tal como manifiesta la copia $n^{\circ} 3$; trozos de estatuas de rica estructura, distinguiéndose en el hombro de una el traje característico de un alto personaje como el Augusto principe que expresa aquella, y otras 3 magnificas que se han recogido en el mismo terreno, a que alcanzaría el templo, dedicado al emperador Augusto; todo su alrededor que se halla sembrado de cimientos de casa, cañerías, arcas de agua, escaleras de piedra, vasijas de barro barnizado de rojo brillante, monedas, y otras mil antigüedades, entre ellas las dos inscripciones, en tabla de piedra una blanquecina y veteada la otra, cuyas copias se unen marcadas con el $n^{\circ} 4$, no dejan dudas que existió la colonia romana de Percejana opulenta que atestigua con otras mil circunstancias la grandeza y lujo con que se encontró algún día.

Un terreno circular y espacioso en dirección E marca el local del circo; innegable es que lo hubo, véase la inscripción $n^{\circ} 5$ "Lucio Valerio Amando y Lucio Valerio Lucumo, cuatorviros, construyeron con el dinero público el podium en el circo, por decoro y honor y decreto de los decuriones"; el Censor que expresa el $n^{\circ} 4$, los cuatrumviros y decuriones funcionarios de importancia, los Valerios que el $1^{\circ}$ de este nombre aparece cónsul en Roma el 244 de su fundación al lado de Junio Bruto y en remplazo de Colatino fundadores de la República; el célebre Q. Marius Cursor cuyo nombre se halla en la inscripción del ara, unido al Julius Amabilis, nombre llevado por emperadores, son bastantes pruebas de los distinguidos patricios que la habitaron y de presumir es que otros infinitos acompañasen a estos cuando a las primeras investigaciones se ha dado con los de estos personajes, nombres salvados milagrosamente de la destrucción del tiempo; contribuye a tener por segura la existencia de Percejana en dicho punto, lo que Sta. Eulalia dice en el martirologio, "Ad locum quid dicebatur Percejana et parentis posesio erat", y el Breviario Romano repite, "Eulalia virgo: in locum quid dicebatur Porcejana in finibus provinciae Betica prope Emeritam miliario trigésimo"; la corta diferencia en que disienten en nada destruyen las apariencias enunciadas pues aun cuando disten mucho los confines de Merida, la Betica alcanzaba tanto mas; la piedra encontrada una legua SO de Medina que dice "terminus augustalis finis Emeritensium", marca los confines de aquella donde se encontraba en el tiempo de Cesar Augusto y Trajano Augusto, y el de la Lusitania en el de Constantino, cuya metrópoli era Mérida. Desde la colonia en dirección de NE partiría una calzada de la que se conservan algunos trozos y a derecha o izquierda en las cañadas contiguas, tropieza el arado todos los días con escombros de casas, trozos de mosaico, vasijas, y otros restos que deja conocer por lo apartado de unos sitios a otros fuesen de casas de campo, más o menos notables que las que aparecen al otro lado del E a media legua; estas con pavimento, las mas, de mosaico, serían construidas a lo largo 
de la vía militar que cruza de Na S: a igual distancia y en igual dirección se encuentra la quinta de Liberio, que aun demuestra con su terreno espacioso, trozos de granito, mármol, lápidas, mosaicos, y otras piedras horadadas y caprichosamente labradas, la grandeza y gusto de sus florecientes dias; en aquellos en que sirvió de refugio a las jóvenes vírgenes, Eulalia y Julia cuando el padre de la primera quiso sustraerlas de la persecución que diezmaba la naciente religión del crucificado; el terreno de esta posesión llamada Ponciano, se conoce en el día por $S^{a}$ Julia o el Palacio y dista 9 y media leguas de Mérida; en 1580 existió una ermita erigida a las dos vírgenes con las inscripciones sepulcrales cuyas copias son marcadas con el $n^{\circ} 6$ y en 1836 fueron recogidas a Medina empero al reedificar las casas Consistoriales las utilizaron en sus paredes con bastante detrimento.

Lastimoso es en verdad que la mano del hombre haya de dar los últimos golpes de destrucción a tan preciosos monumentos. La Comisión conocera la importancia del terreno descripto, la necesidad de poner a salvo las preciosidades que descubra y aumentará otra prueba de la grandeza del suelo de Extremadura que más que ninguna provincia ha podido siempre mostrarse orgullosa.

$\mathbb{1}^{\prime 2}$

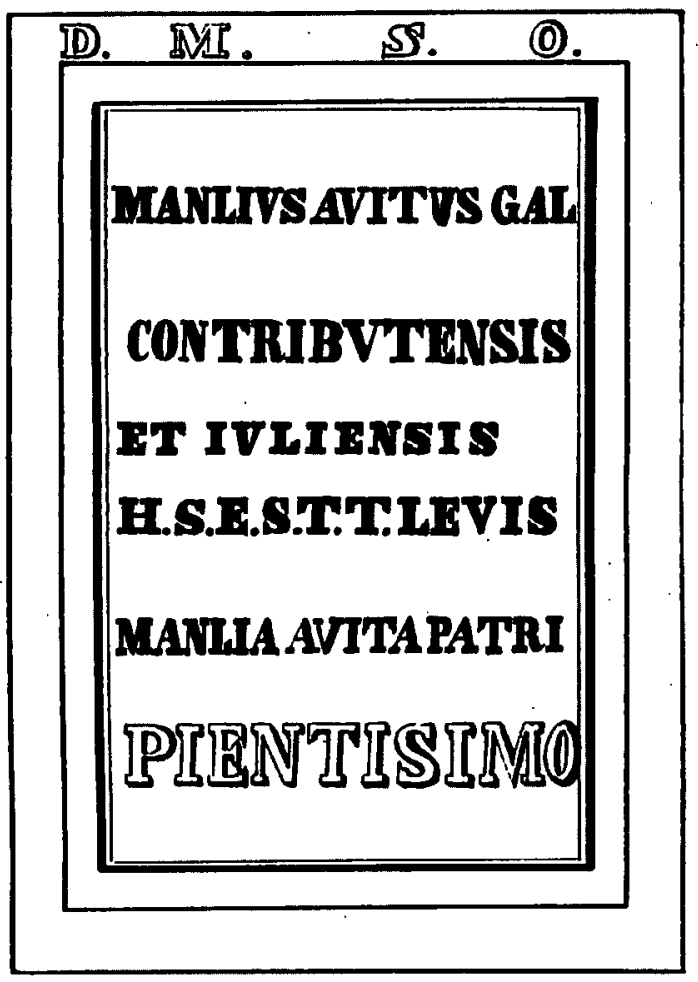

24

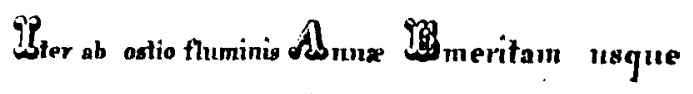

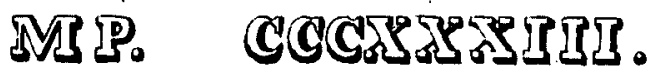

arco 

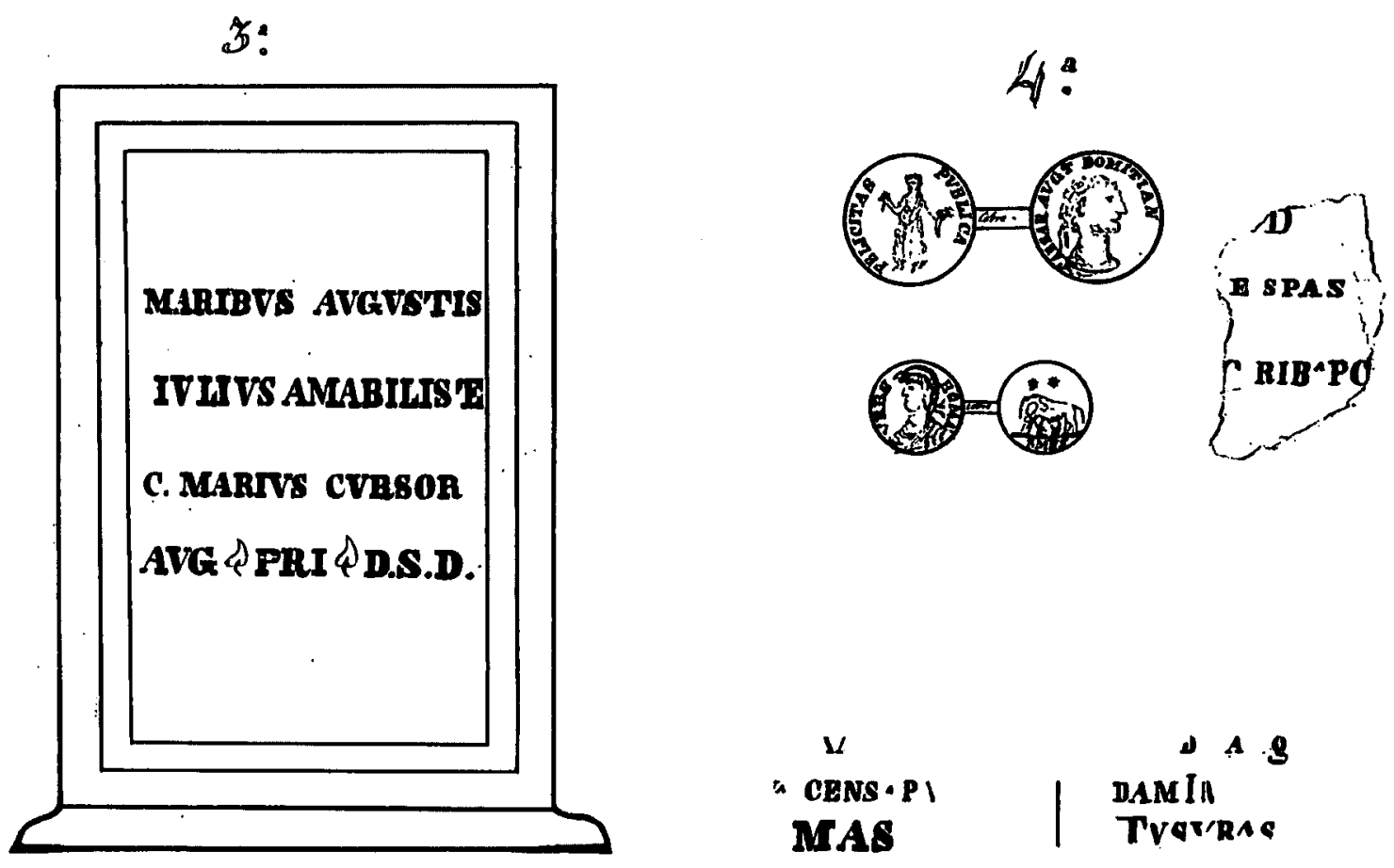

$\mathbf{Y}$

1 CENS • PI

MAS

1 A

DAMIN

Trav'RAs

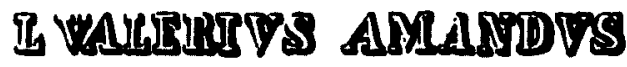

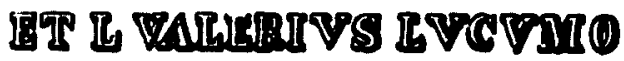

PODIVN IN CIRCO

P DCOR FONORUMI

II P DICRITO

DECVRIONVM

D. \&. P. I. C. 

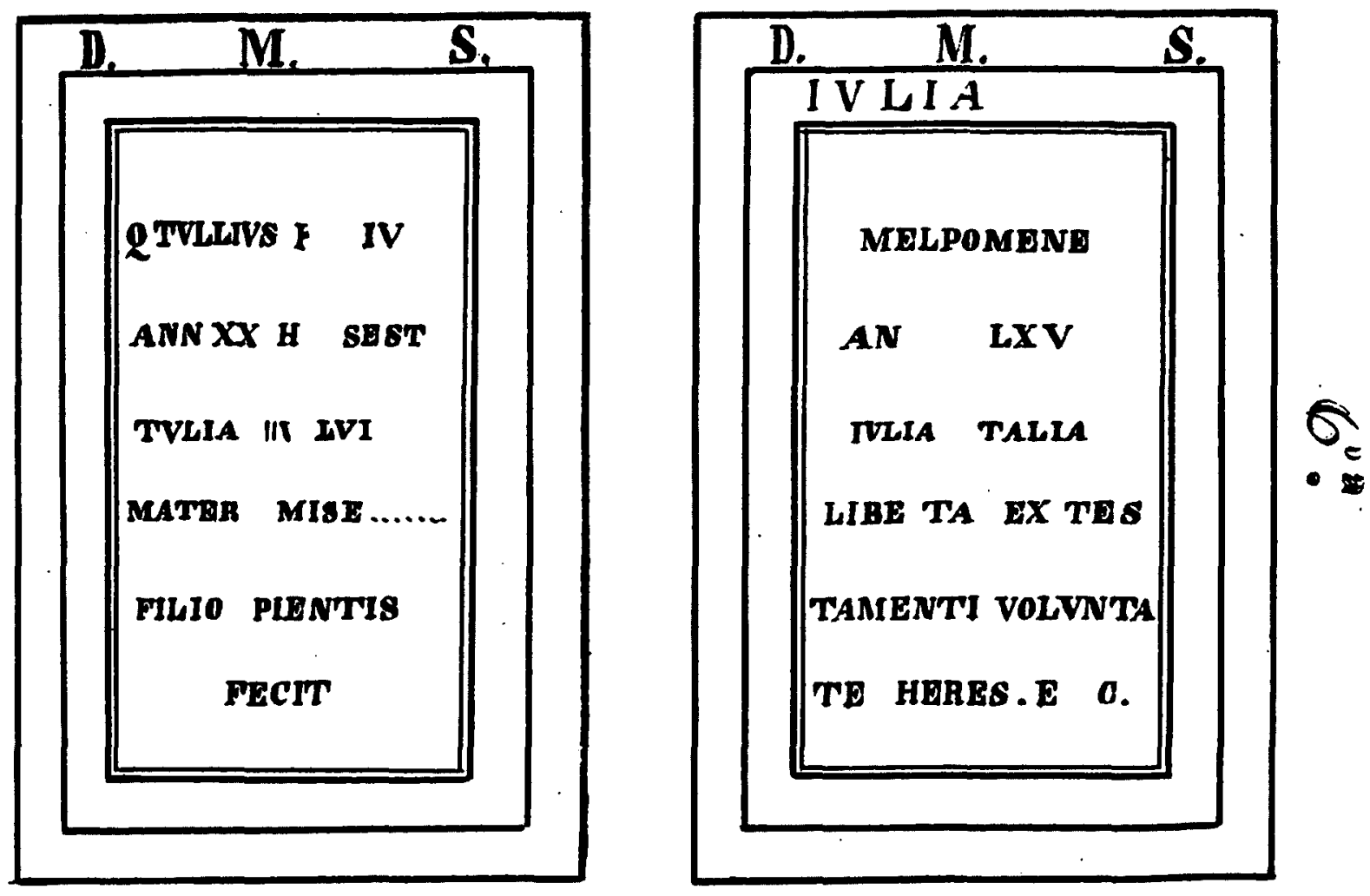

El manuscrito es muy valioso, a pesar de su brevedad, porque aporta una información novedosa en dos aspectos: el arqueológico y el epigráfico.

\section{LA ARQUEOLOGÍA Y LA POLÉMICA SOBRE LA CIUDAD HISPANO-ROMANA QUE EXISTIÓ EN MEDINA DE LAS TORRES}

J. A. Barrientos insiste en la riqueza arqueológica que contiene, y mantiene oculto, el término municipal de Medina de las Torres, del que menciona tres parajes: San Bartolomé el Real, Cerro de San Blas (hoy llamado "Los Cercos") y Santa Julia o El Palacio.

Ciertamente en todos ellos se han hallado inscripciones. Deslumbrado por los hallazgos, Barrientos, intenta identificar la ciudad hispano-romana que produjo tanta riqueza y tercia en la polémica sobre si allí estuvo Contributa Iulia o Perceiana. Aunque conoce y maneja el Itinerario de Antonino, comete el error de no verificar a qué lugar corresponden las distancias, y, por eso, sitúa Perceiana en Medina de las Torres, y muy cerca de ella Contributa Iulia, cuando ambas poblaciones estaban separadas por 20 mil pasos.

En su descargo hay que decir que no dispuso, seguramente, ni del tiempo ni de los datos que en la actualidad ha manejado P. Silliéres, que ha ubicado (con fundamentos sólidos, en nuestra opinión) Contributa Iulia en el paraje de "Los Cercos" (Medina de las Torres) y Perceiana en Villafranca de los Barros (Sillières 1990: 485-487). 
En su favor hay que añadir que, para confirmar su teoría, aporta la documentación epigráfica que él conocía en aquel momento (en parte desconocida hasta hoy), y proporciona además el estado de la cuestión tal como se debatía a mediados del siglo XIX.

En 1634 Rodrigo Caro había ubicado Contributa Iulia "en un despoblado, que se ve a una milla de Fuente de Cantos, que oy llaman S. Bartolomé del Villar" (Caro 1634: 88 y 196), porque ahí coinciden las 44 millas que, según el Itinerario de Antonino, hay desde Emerita a Contributa Iulia. Sin embargo no hizo ninguna precisión sobre Perceiana.

Pero, poco más tarde, Tamayo de Salazar, como Contributa Iulia aparecía -según su lectura- en la inscripción de Manlius Avitus (CIL II 1029; cf. Tamayo 1651-1659, fecha de 10 de diciembre) que se conservaba en la iglesia parroquial de Medina de las Torres, el mencionado autor consideró que esta población badajocense era la betúrica Contributa Iulia.

En el siglo XIX, Ceán Bermúdez seguía la doctrina de Caro y ubicaba Contributa Iulia en S. Bartolomé del Villar, al tiempo que situaba la "Perceiana de los Celtas Betúricos" en Medina de las Torres (Ceán 1832: 281), lo que también afirmaba tres años después Cortés y López ${ }^{5}$, aunque su fundamento científico es totalmente falso e insostenible.

Lamentablemente J. A. Barrientos no explica de quién ha tomado su identificación Perceiana = Medina de las Torres. Parece que bebe de los autores del XIX, pero también conoce la hipótesis de Tamayo. Madoz, como ya hemos visto, sigue identificándola con Perceiana. Sobre Santa Julia y el Palacio o Palacios de Santa Julia dice que "en ellos se conservan lápidas, trozos de columnas y otras antigüedades. Este sitio se designa por algunos como la residencia de $S^{a}$ Olalla antes de su martirio". Madoz no cree que sea aquí sino en Aldehuela (Cáceres) (Madoz 1845-50: XI, 341).

De cualquier manera, el objetivo de Barrientos es interesar a la Comisión de Monumentos para que vele por el rico patrimonio romano de Medina de las Torres e inicie excavaciones sistemáticas. Con este fin aporta pruebas demostrativas de la existencia de una importante ciudad romana. La tradición de ermitas con la advocación de las Santas Julia y Eulalia, y el hallazgo de un "terminus augustalis, finis emeritensium", a una milla de distancia, le parecen pruebas evidentes de que la ciudad es Perceiana.

Es manifiesto que se basa en la tradición del Pasionario Hispánico, según el cual Eulalia se hallaba en una villa, denominada Promtiano, a 38 millas de Emérita, en los límites de la provincia Lusitania con la Bética (Fábregas 1955: 69, IX, 4). La ermita de Santa Julia y Santa Eulalia estaba a 9 leguas y media de Mérida y el terminus augustalis una legua mas al suroeste, 10 leguas y media se corresponden con las 38 millas del pasionario $(57,5 \mathrm{~km}$.), exactamente el lugar donde el padre "quiso contraerlas la Eulalia y a Julia], de la persecución que diezmaba la naciente religión del crucificado", según palabras de Barrientos. El propio Madoz dice que "no lejos de este sitio [los Altos de Sola Parza -donde aparecí́ el terminus augustalis-], están los prados de Santa Julia y cerca el llamado Hornito de Santa Olalla, por lo que es de presumir que el antiguo término de Mérida comprendia estos sitios" (Madoz 1845-50: XV, 454, s.v. Valencia del Ventoso o del Barrial).

Y para corroborarlo, Barrientos incluye las inscripciones halladas en el término municipal de Medina de las Torres, confirmando con ello algo que hemos sabido por otras fuentes, a saber, que el paraje de "Los Cercos", sito a $2 \mathrm{~km}$ del casco urbano, ha proporcionado mucho material de todo tipo, y que concretamente el epigráfico es exponente de la existencia de un municipium hispano-romano ${ }^{6}$.

5. Cortés y López 1835-36; en el volumen III, p. 281, razona así: "Y esta etimología se acerca más a la manifiesta etimología de esta ciudad, que sale de la voz griega Pyrgos, que significa 'torre o castillo', y en este concepto Perceiana o Pergelana significa 'ciudad de las torres'". Evidentemente Pergelana, seguramente forma corrupta del Ravenate, no tiene ninguna relación etimológica con Pyrgos, pero la idea la volveremos a encontrar en Madoz (1845-50: XI, 341, s.v. Medina de las Torres).

6. Cf. EE VIII, 90 (epitafio de un séviro augustal); CIL II 1025 (con dedicatoria seguramente al municipium); y las inéditas contenidas en este manuscrito. 


\section{EL REPERTORIO EPIGRÁFICO}

\section{1.- El término augustal de Valencia del Ventoso.}

José Antonio Barrientos fue el primero, cronológicamente hablando, que dio noticia del terminus augustalis hallado en Valencia del Ventoso ${ }^{7}$ con la siguiente lectura sin división de líneas: terminus augustalis finis Emeritensium. No da las referencias concretas sobre el paraje, circunstancias, etc., pero el texto y la ubicación permiten identificarlo sin ninguna duda.

Con su referencia disponemos de un nuevo dato para zanjar definitivamente la polémica que originó una lectura deficiente de Fita, al leer como terminus augustalis una inscripción hallada en la finca dehesa de "El Santo" 8 , y que fue después identificada erróneamente por Álvarez Sáenz de Buruaga (1976: 23), con el terminus que Barrientos menciona en este manuscrito, el cual está depositado, y conservado, en el MAP de Badajoz y en el que recientemente ${ }^{9}$ se han leído, al final, unas letras, que ni Barrientos ni todos los que lo leyeron después vieron, como tampoco nosotros hemos visto. El ms. de J. A. Barrientos coincide, en texto y procedencia, con lo que dijeron Madoz (XV,454), Del Solar (s/a: $\left.n^{\circ} 42\right)$, Álvarez Martínez (1988: 185-92) y Ramírez Sádaba (1993: 301-5). J. A. Barrientos precisa que el terminus se halló una legua al suroeste de Medina de las Torres, referencia que se aviene grosso modo con el paraje "Los Altos de Sola Parza" de Valencia del Ventoso. Por el contrario la dehesa de "El Santo" de Montemolín está al sureste, a más de 5 leguas de distancia.

\section{2.- Las inscripciones de Medina de las Torres}

El manuscrito enriquece nuestros conocimientos sobre la epigrafía procedente de Medina de las Torres y nos afianza en la hipótesis de que Contributa Iulia estuviera en el paraje de "Los Cercos". Se menciona el lugar de hallazgo de algunas inscripciones, circunstancia que hasta ahora desconocíamos, y proporciona, además, noticia de otras, que han desaparecido, y de las que no se tenía constancia. Veámoslo de manera sucesiva.

\subsection{EI epitafio de Q. Manlius Avitus (CIL II: 1029), hoy perdido.}

Hasta este momento disponíamos de tres traditiones. Según Caro se hallaba "en el término de Zafra" y, según Masdeu, "en Medina de las Torres" (sin más precisión). Únicamente Tamayo de Salazar la situaba "en la pared de la iglesia parroquial de Medina de las Torres", cosa que queda corroborada por Barrientos, quien precisa también que la pieza procede de San Bartolomé el Real y que en 1845 ya no se podía leer.

Según el manuscrito en 1824 se sacó una copia (la de Boxoyo probablemente) que, al parecer, vio J. A. Barrientos. Sin embargo, resulta sorprendente que repite la misma traditio (exactamente la misma) que

7. La publicación más reciente en la que se recogen las anteriores es Salas y otros 1997: 64, $\mathrm{n}^{\circ} 43$.

8. La inscripción ha reaparecido recientemente. Es un fragmento de placa de mármol con un texto honorífico y no un término augustal según González 1996: 84-85, $n^{\circ}$ I, empotrado en una pared de la casa de labor de la finca de la Dehesa de El Santo, una parte de cuya extensión cae en el término municipal de Montemolín (Badajoz) y otra en el de El Real de la Jara (Sevilla). Su texto, según

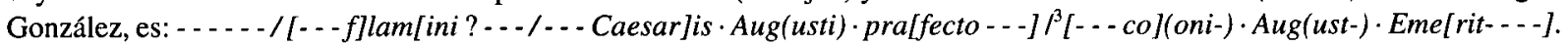

9. Ingresó en dicho museo en 1924. El texto es : Terminus Augu/stalis finis E/meritensium. Canto (1995:317), para su hipótesis sobre la localización de Siarum Fortunale, considera de nuevo este hito como del territorio de Montemolín y propone la siguiente lectura: Termînus $\cdot$ Auglu]/stalis $\cdot$ f[in]ils E]m/eri[t]ensis $\cdot$ in $[t]$ [ [r] /S(iarenses) $\cdot$ For[tun]ale[s]. La misma es repetida en la reciente obra de dicha autora, Canto 1997: ${ }^{\circ} 153$. Pero después de Emeritensium no es visible ningún vestigio de letras, como ya dice A.U. Stylow en su comentario en $\operatorname{HEp} 5,1995, \mathrm{n}^{\circ} 115$. 
da Tamayo. Y en esta coincidencia resulta extraño que en el coronamiento haya una $\mathrm{O}$ tras la fórmula DMS. Más lógico es pensar que se trata de la Q. del praenomen, que seguramente Tamayo no entendió y que, por su condición de abreviatura alineó con las precedentes, desvinculándola así del nombre al que pertenecía. Así se explicaría que Tamayo dé una primera línea D M S O, y a continuación el nombre y la tribus, texto cuya ordinatio tampoco concuerda con la que dan los otros autores ni con la que acepta Hübner.

La inscripción debía estar especialmente machacada en 1.4 como lo demuestra la interpolación de Tamayo a partir del texto de Plinio $(3,14)$ y de los pocos restos de las letras que quedaban, los cuales, según Boxoyo, eran ...VII · P B BIS · AN...VI. Tamayo ni siquiera debió leer nada después de AN, imaginando ET IVLIENSIS por lo que quedaba de la raya horizontal del numeral y con el primer I de la cifra, de donde ET. Con los trazos que seguían correspondientes a IIVIR BIS, e intercalando una $\mathrm{N}$ entre los restos de la panza de la $\mathrm{R}$ (para él los trazos horizontales de una $\mathrm{E}$ ) y los trazos curvos de la $\mathrm{B}$ (para él una S), inventó IVLIENSIS.

Como la copia de 1824 (probablemente la que publica Masdeu que la había recibido de Boxoyo) no mencionaba Contributa Iulia, posiblemente Barrientos (o alguien antes que él) a quien lo que le interesaba era ubicar los antiguos lugares de Contributa Iulia y Perceiana debió preferir la lectura de Tamayo por citarse en ella a los Contributenses Iulienses y así combinó ambas lecturas. Al margen de los errores derivados de esta nueva interpretación de las dos copias, lo que queda evidentemente claro es que la pieza era medinense, subsanándose así las imprecisiones de Caro y Masdeu y, como hemos visto, se explica por el mal estado del texto la diferente traditio que Boxoyo proporcionó (poco antes de 1824) a Masdeu.

\subsection{La inscripción honorífica de los seviros L. Valerius Amandus y L. Valerius Lucumo (CIL II: 984).}

Hoy perdida, la traditio y, sobre todo, la ubicación que nos da Barrientos es sumamente interesante. Según su dibujo es clara la ligatura DE, que confirmaría la lectura de Caro, DEC y por tanto parece que debe ser aceptada.

En nuestra opinión, debemos dar crédito a Barrientos, porque, como se verá a continuación, transcribe lo que ve, aunque no lo entienda. Así, en la línea 4 no entiende la grafía del sevirato y la traduce por quatuorviros, pero transcribe fielmente lo que ve. Lo mismo ocurre en la línea 7, donde copia I lo que es F.

Por eso en la línea 4 entiende DECOR y traduce "por decoro y honor", sin percatarse de que la sintaxis latina hubiera requerido la preposición $\mathrm{OB}$, pero nunca $\mathrm{P}(\mathrm{ER})$ como él entiende y traduce.

En cuanto a la ubicación, según Constanzo (véase CIL II) esta inscripción estaba en un lugar llamado Palenciana (hoy Las Palencianas) que está muy próximo al de El Palacio. La autopsia de la inscripción de Barrientos y su inclusión entre las de Medina de las Torres confirma la localización de Constanzo y Masdeu en este término y no en el de Zafra que dio Caro. La indicación "Zafra" que da este último debe ser manejada con cautela. Muchas veces es una referencia vaga a un término amplio. Así la vemos utilizada en la procedencia de Caro de la inscripción del IIvir de Medina de las Torres que se encontraba empotrada en la parroquia (CIL II: 1029) o en otra de Alconera (CIL II: 997).

\subsection{Inscripciones de Q. Tullius [- - - y de Iulia Melpomene.}

Ambas las había visto Monsalud (1898: 471-175) (su primer editor) en el mismo lugar en que se conservan, en el Ayuntamiento de la localidad. Pero ignorábamos su procedencia. Barrientos dice que proceden de "una ermita erigida a las dos vírgenes [Eulalia y Julia] ... y en 1836 fueron recogidas en Medina, empero al edificar las casas Consistoriales las utilizaron en sus paredes con bastante detrimento".

Por tanto sabemos que en el paraje denominado "Santa Julia o El Palacio" existía una ermita (y tal vez subsista un yacimiento arqueológico). Ya hemos visto que de un lugar muy próximo, "Las Palencianas", 
procede la inscripción de los seviros. Todo ello indica que, a cierta distancia de "Los Cercos" (5 km aproximadamente) había también construcciones antiguas o que reutilizaron materiales antiguos.

Si cotejamos el texto del manuscrito con el de Monsalud, obtendremos algunas aclaraciones de lectura.

Monsalud
D M S
Q TVLLIVS HIL[---]
[A]NN XXI H S ES[T]
TVLLIA [ ]STVI[---]
MATER EX TES[T]
FILIO PIENTI[SS]
FECIT

\author{
Barrientos \\ D M S \\ Q TVLLIVS + IV \\ ANN XX H SEST \\ TVLIA $+\backslash$ LVI \\ MATER MISE[---] \\ FILIO PIENTIS \\ FECIT
}

V. 2. Hoy solo se aprecia un hasta recta, inmediatamente después de TVLLIVS. Corresponde al signo indeterminado que dibuja Barrientos.

V. 4, Hoy se aprecia IV. El hasta oblicua que, según Barrientos, precede, hoy parece una raspadura, pero efectivamente es trazo de hasta; delante se aprecian restos de $S$, que vieron también Monsalud y Wickert. Se puede leer SALVI[-] y se puede proponer un cognomen del tipo SALVIA, SALVILLA o SALVIOLA. Pero como el espacio que queda es pequeño, lo más posible es SALVIA.

V. 5. Parece más acertada la lectura MISE de Barrientos.

Debemos renunciar a ver un cognomen del tipo Hilarus o similar, así como la razón testamentaria para colocar el ara. Podemos razonar que comenzaría por P o F y terminaría en IVS. Conviene observar que la separación que Barrientos hace en su dibujo no es signo inequívoco de que falten letras. En la línea siguiente, HSEST no hay ninguna laguna. Con esta evidencia podría leerse una palabra corta quizá PIVS, como cognomen de TVLLIVS.

En la línea 6 habría que leer MATER MISE[RA] o MISE[RRIMA].

Algo similar puede decirse de la otra inscripción. Por el fragmento visible en la pared creíamos que se trataba de una placa. El dibujo de Barrientos permitiría suponer que puede ser un ara, puesto que es similar al de la pieza anterior. Queda la duda de la moldura, que es la misma en ambos casos, y que no existe en el caso de la inscripción de Q. Tullius. Pero es evidente que esta pieza ha sido repicada, por lo que pudo tener también moldura. De todos modos la fórmula DMS no pudo ocupar la misma posición en ambas piezas: en la de $\mathrm{Q}$. Tullius está debajo de la moldura, mientras en la de Iulia Talia tuvo que grabarse por encima (en el coronamiento ?).

Lo más importante es que en el exemplum de Barrientos se añaden dos líneas que en la pieza actual no se ven. Y también transcribe completa la última línea, cosa que ni vio Monsalud ni se ve en la actualidad. Cotejemos ambas traditiones: 


Monsalud
MELPOMENE
AN LXV
IVLIA TALIA
[ ]IBETA EX TES
[---]ENTI VOLVNTA
TE F C

\author{
Barrientos \\ D M S \\ IVLIA \\ MELPOMENE \\ AN LXV \\ IVLIA TALIA \\ LIBE TA EX TES \\ TAMENTI VOLVNTA \\ TE HERES E C
}

El cotejo sugiere dar crédito a Barrientos. Lo que hoy podemos leer (y lo mismo le ocurrió a Monsalud) es que Melpómene es la patrona de Julia Talía. Y hasta ahora así se había copiado y recopiado la pieza. Nadie había sugerido que podía faltar al menos una línea, puesto que la patrona aparecía denominada únicamente por el cognomen, cosa anómala, máxime cuando la propia liberta aparece claramente denominada Iulia Talia ${ }^{10}$.

Evidentemente, la moldura parecía desaconsejar la hipótesis de una línea anterior a la de Melpomene pero el dibujo de Barrientos indica claramente que Iulia se grabó en la cornisa, cosa que alguna vez se encuentra en la propia Beturia (como el ara de Iulia Felicissima en Jerez de los Caballeros ${ }^{11}$ ).

Y, siguiendo la misma lógica, debemos pensar que la fórmula DMS estaba en el coronamiento.

Con la lectura de Barrientos la pieza se comprende mucho mejor. Iulia Talia era liberta de Iulia Melpomene, y ésta es la única denominación correcta. Además era su heredera, y, efectivamente, HERES ocupa exactamente el mismo espacio que MENTI, puesto que puede apreciarse perfectamente que la $\mathrm{F}$ está debajo de la primera $\mathrm{V}$ de VOLVNTA.

Se puede añadir en favor de la credibilidad de Barrientos que transcribe E en vez de F. Es evidente que no entendió bien la fórmula final, y copió lo que vio.

\subsection{Inscripciones inéditas.}

Su hallazgo en la colina de "Los Cercos" confirma que aquí está el yacimiento de especial importancia y que, por la calidad y la tipología de sus epígrafes, tuvo que ser el centro urbano del municipium. De aquí proceden al menos cinco de las inscripciones conocidas, entre ellas la imperial, la votiva y una funeraria que contiene el epitafio del sevir augustal, como ya se ha dicho. Por tanto, con estos nuevos documentos casi se puede asegurar que en "Los Cercos" estuvo Contributa Iulia Ugultunia, como supusieron Galsterer (1971: 20s., nota 39), Rodríguez - Ríos (1976: 151), Sillières (1990: 476s., 485 y 487s.) y Fear (1991: 153).

$1^{12}$. Pedestal con el campo epigráfico enmarcado por una moldura. Le falta el coronamiento. En la última línea dos hederae. Se halló en la colina de San Blas (hoy Los Cercos). Perdida.

10. Para Canto (1997 $\mathrm{n}^{\circ}$ 127), "la primera impresión, dado que ambas llevan cognomina de musas, lleva a creer que eran esclavas de un lulius y que con la 'herencia' de Melpómene, Talía pudo comprar su libertad y dedicar a su ihermana, amiga? este monumento funerario". Ahora se sabe, por el texto de Barrientos, que Talía era esclava de Melpómene y recibió la libertad por manumision testamentaria (ex testamenti voluntate).

11. Cf. Naval (1930: 842-843)

12. Delgado (1998: 269) dio una primera noticia de la inscripción. 
MARIBVS AVGVSTIS

\author{
[- ?] IVLIVS AMABILIS ÊT \\ C(aius) MARIVS CVRSOR \\ AVG(ustales) $\bullet$ PRI(mi) $\bullet \mathrm{D}(\mathrm{e}) \cdot \mathrm{S}($ uo $) \cdot \mathrm{D}$ (ederunt) $\cdot$
}

El hecho de que la primera palabra de 1.1 carezca de sentido en este contexto nos hace pensar que lo que en realidad hubiera estado escrito es Matribus con un nexo TR como el que hay al final de línea 2, de forma que el monumento habría sido dedicado por los augustales [- ?] Iulius Amabilis y C. Marius Cursor a las Matres Augustas. Es el primer testimonio de culto a las Matres en la Beturia, culto de tradición indoeuropea escasamente documentado fuera de la Península Ibérica. No es extraño hallarlo en la Beturia Céltica. Quizá lo más sorprendente es que se trata de la primera dedicación a las Matres Augustae en Hispania ${ }^{13}$.

Respecto a la indicación primi $i^{14}$ podría entenderse como que se trata de los primeros seviri del municipio antes que pensar en que son los primeros seviros del municipio que realizan esta donación como acto evergético ${ }^{15}$ o en una posible organización jerárquica interna de los Seviri ${ }^{16}$.

La mala lectura maribus por matribus y la excepcionalidad del culto a las matres Augustas en esta área podrían hacer pensar que pudiera tratarse de un falso fabricado ex novo o de una inscripción de otro lugar que se haya atribuido a éste. También habíamos considerado la posibilidad de que se tratase de un pedestal a los Laribus Augustis. Sin embargo Barrientos no parece distorsionar lo que ve y el propio hecho de que ponga maribus indica su falta de conocimiento de la epigrafía romana y por ello pensamos, como hemos comprobado también para las inscripciones anteriores, que debemos otorgarle crédito. También es posible que la carencia de praenomen del primer augustal no sea tal sino que no lo hubiera leído, aunque realmente tampoco sería extraño que no lo hubiera tenido. A favor de su autenticidad está también el título de augustalis, a secas, habitual en el Conventus Hispalensis (Delgado 1998: 269).

Aceptando que la inscripción es auténtica, tenemos un nuevo dato para la municipalidad del yacimiento situado en los Cercos de donde ya se conocía un IIvir (CIL II: 1029) y la existencia del sevirato pues como ya hemos visto la inscripción que Caro atribuye a Zafra con la donación de un circo (CIL II: 984) hay que situarla en Medina.

Si se tratara de los primeros seviros del municipio cabría pensar en una fecha en el siglo I avanzado.

2. Fragmento de una placa probablemente de mármol blanco (una blanquecina). Interpunción triangular. Se halló en la "Colina de San Blas":

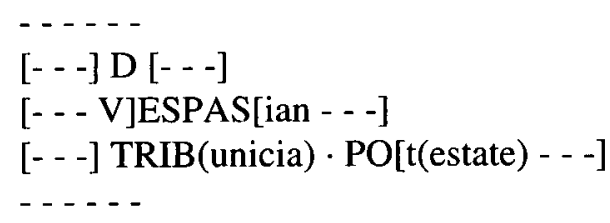

13. Matres Aufaniae en Carmona (AE 1968: 226); véase también Gómez-Pantoja 1999: 421-432; en 423 hace referencia a esta inscripción, aunque da la procedencia equivocada de Regina. Sobre las Matres en general en las provincias occidentales véase Rüger 1987: 1-30.

14. En Hispania aparece la palabra primus asociada también a un seviraug. en Dertosa (CIL II $\left.{ }^{2} / 14,795\right)$ y a otro en Suel (CIL II 1944) cf. Arrizabalaga 1994: 258-259, 263.

15. Sobre la utilización de la expresión primus omnium en relación con actos evergéticos véase Mrozek 1971, 66-69.

16. Sobre la mención primi de los seviri de CIL VI 29681 de Trebula Suffenatium y la organización de los Augustales véase Abramenko 1991, 592 ss. y Abramenko 1993: 13ss. 
CEÁN BERMÚDEZ, J.A. (1832): Sumario de las antigüedades romanas que hay en España, en especial las pertenecientes a las Bellas Artes, Madrid.

CORTÉS Y LÓPEZ, (1835-36): Diccionario histórico-geográfico de la Edad Antigua: Tarraconense, Bética y Lusitania, Madrid.

DELGADO DELGADO, J.A. (1998): Élites y organización de la religión en las provincias romanas de la Bética y las Mauritanias: sacerdotes y sacerdocios, BAR International Series 728.

DEL SOLAR, A. (s/a): Libro de Registro del MAP de Badajoz, Serie Greco-Romana.

FÁBREGAS GRAU, A. (1955): Pasionario Hispánico, Vol. II, Madrid-Barcelona.

FEAR, A.T. (191): "Contributa Iulia, Ugultunia and Curiga", Gerión 9: 151 ss.

GALSTERER, H. (1971): Untersuchungen zum römischen Städtewesen aufder Iberischen Halbinsel, Berlin.

GIMENO, H. (1999): "Regina: algunos datos epigráficos inéditos en un manuscrito de José Barrientos (1845)", Sylloge Epigraphica Barcinonensis 3 (Cornucopia 6) (en prensa).

GÓMEZ-PANTOJA, J. (1999): "Las madres de Clunia", Pueblos, lenguas y escrituras en la Hispania Prerromana, Salamanca: 421-432.

GONZÁLEZ FERNÁNDEZ, J. (1996): “Mansio Mons Mariorum (It. Ant. 432.4)”, Habis 27: 83-95.

MADOZ,P. (1845-50): Diccionario geográfico-estadístico-histórico de España y sus posesiones de ultramar, Madrid.

MONSALUD, Marqués de (1898): "Epigrafía romana de Medina de las Torres y Fregenal de la Sierra", BAHist. 32: 471-475.

MROZEK, M. (1971): “Primus Omnium sur les inscriptions des Municipes Italiens", Epigraphica 33: 60-69. NAVAL (1930): Nuevas aras funerarias en Jerez de los Caballeros, BAHist., 836-845.

RAMÍREZ SÁDABA, J.L. (1993): "Dos Termini augustales del territorium emeritense: uno de Valencia del Ventoso y otro de Montemolín", Veleia 10: 301-305.

RODRÍGUEZ BORDALLO, A; RÍOS GRAÑA, A.M. (1976): "Contributa Iulia Ugultuniacum”, V Congreso de Estudios Extremeños, Badajoz 1976.

RÜGER,C.B. (1987): "Beobachtungen zu den epigraphiscen Belegen der Muttergottheiten in den lateinischen Provinzen des Imperium Romanum", Matronen und verwandte Gottheiten, Köln - Bonn (Beihefte der BJ 44).

SALAS MARTÍN, J. Y OTROS (1997): Inscripciones romanas y cristianas del Museo Arqueológico Provincial de Badajoz, Badajoz.

SILLIÈRES, P. (1990): Les voies de communication de l'Hispanie meridionale, Paris.

TAMAYO DE SALAZAR (1651-59): Martyrologium Hispanum. Anamnesis sive commemoratio omnium ss. Hispanorum... ad ordinem martyrologii, Lugduni, fecha de 10 de Diciembre. 